\title{
Enhanced microwave transmission through quasicrystal hole arrays
}

\author{
N. Papasimakis, ${ }^{\text {a) }}$ V. A. Fedotov, A. S. Schwanecke, and N. I. Zheludev \\ Optoelectronics Research Centre, University of Southampton, Southampton SO17 1BJ, United Kingdom \\ F. J. García de Abajo \\ Instituto de Óptica, CSIC, Serrano 121, 28006 Madrid, Spain
}

(Received 19 April 2007; accepted 30 July 2007; published online 23 August 2007)

\begin{abstract}
The authors report on the observation of enhanced microwave transmission through quasiperiodic hole arrays in metal films. The fraction of transmitted light reaches $50 \%$ in a self-standing metal film and approaches $90 \%$ when the film is sandwiched between thin dielectric slabs, while the holes occupy only $10 \%$ of the sample area. The maximum transmission exhibits a Breit-Wigner resonance behavior, accompanied by zero phase change and rendering the film almost invisible over a wide frequency range. The extraordinary transmission phenomenon is interpreted in terms of resonances in the self-consistent interaction between holes, which are represented by effective electric and magnetic dipoles. (C) 2007 American Institute of Physics. [DOI: 10.1063/1.2773763]
\end{abstract}

Little hope of having large light transmission through small subwavelength apertures was allowed by the pioneering work of Bethe, who predicted a drop in the intensity transmitted through a single hole of radius $r$ in a thin perfectconductor screen as $(r / \lambda)^{4}$ for large wavelength $\lambda \gg r$. ${ }^{1}$ However, the situation changed drastically when periodic arrays of apertures were considered rather than isolated holes. Although hole arrays were extensively studied as artificial dielectrics in the past, ${ }^{2}$ the interest in this phenomenon was recently renewed by the work of Ebbesen et al., ${ }^{3}$ who demonstrated experimentally that the optical transmission through subwavelength hole arrays on metal films can exceed by several orders of magnitude the original predictions by Bethe. The periodicity of the arrays was central in the explanation of enhanced transmission both in plasmonic metals ${ }^{4,5}$ and in perfect conductors, ${ }^{6}$ the latter being relevant in observations of the effect at terahertz $z^{7,8}$ and gigahertz $z^{9,10}$ frequencies. However, extraordinary transmission was also observed in quasiperiodic hole arrays in the optical ${ }^{11-14}$ and terahert $\mathrm{z}^{15}$ regimes. The origin of the phenomenon was traced back to the interaction of surface plasmons with Bragg peaks in the reciprocal space of the array, ${ }^{11,14}$ whereas similar results were predicted also for perfect conductors. ${ }^{16}$

It is known that a single hole on a thin ${ }^{1}$ or a thick ${ }^{17}$ metal film can be represented by a magnetic dipole parallel to the surface and an electric dipole perpendicular to it. In the case of a hole array, the collective response admits a representation in terms of the self-consistent polarization $\mathbf{p}_{\mathbf{R}}$ of each hole at the positions $\mathbf{R}$ in response to an external field $\mathbf{E}^{\text {ext }}$ plus the field induced by other holes $\mathbf{R}^{\prime} \neq \mathbf{R}$ via the hole polarizability $\alpha$, that is,

$$
\mathbf{p}_{\mathbf{R}}=\alpha\left[E^{\mathrm{ext}}(\mathbf{R})+\sum_{\mathbf{R}^{\prime} \neq \mathbf{R}} G\left(\mathbf{R}-\mathbf{R}^{\prime}\right) \mathbf{p}_{\mathbf{R}^{\prime}}\right],
$$

where $G\left(\mathbf{R}-\mathbf{R}^{\prime}\right)$ describes the field produced at hole $\mathbf{R}$ by the polarization of the hole at $\mathbf{R}^{\prime}$. In the small hole limit ( $\lambda \gg r$, where $r$ is the hole radius), we can retain only the dipolar component of p. ${ }^{17}$ By considering an incident plane wave with $\mathbf{k}_{\|}$momentum parallel to the film and assuming a

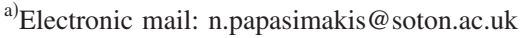

$\exp \left(i \mathbf{k}_{\|} \cdot \mathbf{R}\right)$ spatial dependence for the hole polarizability, ${ }^{18}$ Eq. (1) can be rewritten as

$$
\mathbf{p}_{\mathbf{R}} \approx \frac{1}{1 / \alpha-G_{\mathbf{k}_{\|}}} E^{\mathrm{ext}}(\mathbf{R}),
$$

where $G_{\mathbf{k}_{\|}}=\sum_{\mathbf{R} \neq 0} G(\mathbf{R}) e^{-i \mathbf{k}_{\|} \cdot \mathbf{R}}$ is the sum of the dipole-dipole interaction over the quasilattice. Finally, the transmission $T$ is given by the coherent superposition of the far field produced by all induced dipoles, or equivalently, the transmission along a direction defined by a projected parallel momentum $\mathbf{k}_{\|}^{\text {out }}$ is the far field produced by the dipole,

$$
\sum_{\mathbf{R}} \mathbf{p}_{\mathbf{R}} e^{-i \mathbf{k}_{\|}^{\text {out }} \cdot \mathbf{R}}
$$

The lattice sum $G_{\mathbf{k}_{\|}}$exhibits pronounced maxima when the main diffraction peaks become grazing, which are the equivalent of the Wood anomaly condition in quasicrystal arrays. According to Eq. (2), the transmission will actually exhibit a dip at the divergences of $G_{\mathbf{k}_{\|}}$and a maximum signaled by the minimum of $\left|1 / \alpha-G_{\mathbf{k}_{\|}}\right|$.

In order to further investigate the extraordinary transmission mechanism, we consider a quasiperiodic pattern consisting of 313 circular holes of radius $r=0.46 \mathrm{~cm}$. The side of the repeated basic units of the array is $d=2.31 \mathrm{~cm}$ [see Fig. 1(a)]. In Fig. 1(b), the reciprocal space of the quasicrystal is

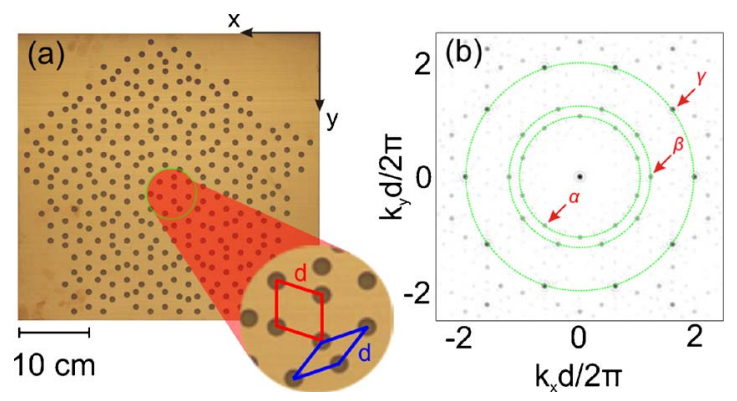

FIG. 1. (Color online) (a) Quasiperiodic hole array drilled in a copper film deposited on a dielectric substrate. The basic units of the quasicrystal are two rhombi with side length $d=2.31 \mathrm{~cm}$ (inset). (b) Fourier transform of the quasiperiodic pattern normalized to $d$. The three strongest Fourier maxima $(\alpha, \beta$, and $\gamma)$ are contained in the corresponding green rings. 

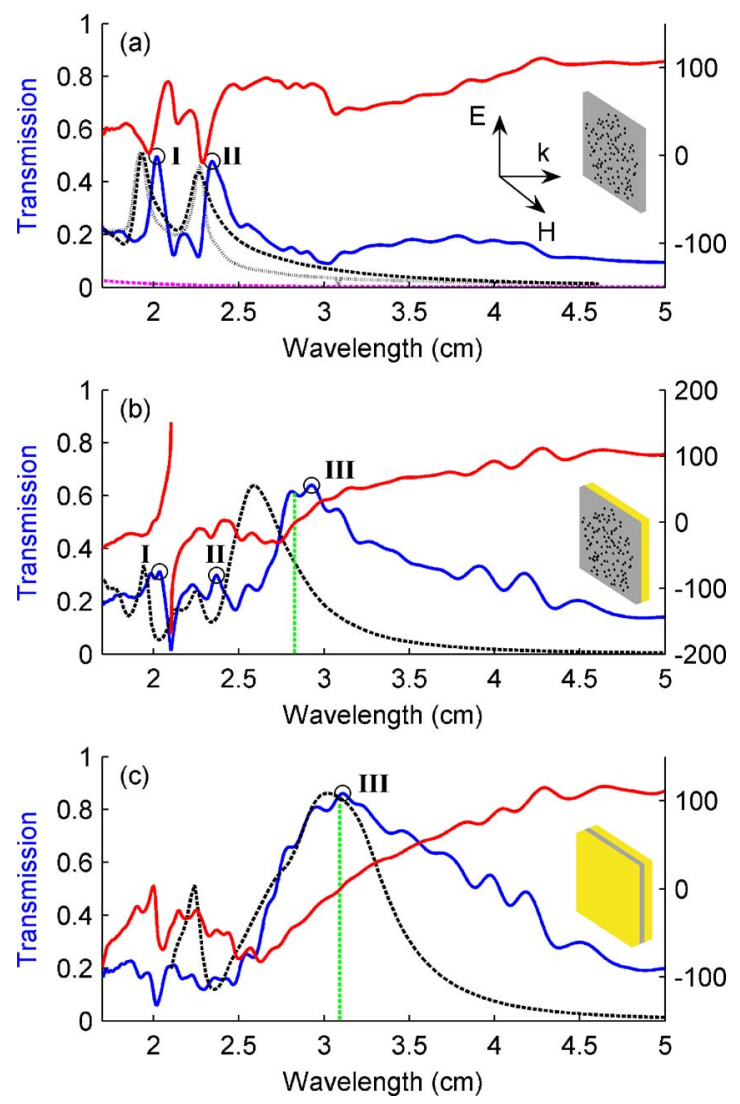

FIG. 2. (Color online) Normal incidence transmission spectra through quasicrystal hole arrays on (a) a self-standing $\mathrm{Al}$ film, (b) a copper film supported by a dielectric substrate, and (c) a copper film sandwiched between two identical dielectric slabs. The Bethe prediction is shown by the dashed purple line in (a), while the green lines in (b) and (c) mark the wavelength positions of invisible metal states, where high transmission is accompanied by zero phase change. Experimental results (solid curves) are compared with theoretical calculations obtained in the coherent-phase approximation [Eqs. (2) and (3)] (dashed curves) (Ref. 19). The latter are normalized to match the maximum of the corresponding experimental curves. The solution of the full dipole representation of Eq. (1) is also offered in (a) (gray dotted curve). Experimental results include both transmittance (blue soild curves, vertical left scale) and phase change after transmission (red solid curves, vertical right scale in degrees).

also shown, where it can be seen that it is composed by dense Bragg peaks and exhibits high orientational order. Although a very large number of peaks is visible, three very strong Fourier maxima can be distinguished $(\alpha, \beta$, and $\gamma)$, located at the circumference of circles with dimensionless radii equal to $1.05(\alpha), 1.23(\beta)$, and $1.98(\gamma)$, corresponding to spatial periods of $2.20,1.88$, and $1.17 \mathrm{~cm}$, respectively. The relation between the diffraction and transmission peaks becomes apparent in the calculated transmission spectra presented in Fig. 2(a), using the coherent-phase approximation (dashed curve) and the full solution of Eq. (1) (gray dotted curve). Both calculations coincide reasonably well, thus justifying the coherent-phase approximation. ${ }^{18}$ Moreover, two transmission maxima are predicted at 1.92 (I) and $2.27 \mathrm{~cm}$ (II), corresponding to the two lowest frequency Fourier maxima of the quasicrystal ( $\beta$ and $\alpha$, respectively).

The quasiperiodic pattern described above was used to manufacture two different samples of $44 \times 46 \mathrm{~cm}^{2}$ overall size: a self-standing aluminum film of $0.5 \mathrm{~mm}$ thickness and a $35 \mu \mathrm{m}$ copper film residing on a $1.5 \mathrm{~mm}$ thick dielectric substrate [see Fig. 1(a)]. Although different materials and thicknesses were used for the two samples, both metals be- have as nearly perfect conductors at the frequency range of interest, while we have confirmed numerically that the difference in thickness $(\ll \lambda)$ has only minor effects on the performance of the arrays. Our microwave measurements were performed in the range of $2-18 \mathrm{GHz}$, in an anechoic chamber using a vector network analyzer and two horn antennas. The sample was placed between the antennas and the transmitted intensity and phase at normal incidence were recorded.

The experimental results for the polarization along the $y$ axis [see Fig. 1(a)] are shown in Fig. 2 (solid curves) and are normalized only to transmission through free space. For the orthogonal polarization, similar (although not identical) results were obtained and are therefore omitted. For comparison, we have also included theoretical predictions derived in the coherent-phase approximation of Eqs. (2) and (3). In all cases, the transmission through the hole arrays exceeds Bethe's predictions, since about approximately ten times more intensity than what is directly incident in the area occupied by holes is transmitted. In particular, for the self-standing array [Fig. 2(a)], two sharp transmission peaks can be seen at $2.02 \mathrm{~cm}$ (I) and $2.34 \mathrm{~cm}$ (II) wavelengths on a slowly decaying transmission background, which as expected, lie very close to the positions predicted by theoretical curves in the same figure. Peaks of shorter wavelength arising from lower spatial frequencies (e.g., $\gamma$ ) lie below the measured frequency range. The width of the peaks is primarily controlled by the number of holes on the sample, since, given the manufacturing accuracy, broadening effects due to fabrication imperfections are unlikely. The magnitude of the peaks is $50 \%$ and $48 \%$, respectively, while at the same time the phase change of the transmitted wave for both peaks is close to zero.

The situation changes dramatically when the metal film is supported by a dielectric substrate [Fig. 2(b)]. Peaks I and II become considerably weaker (30\% and $31 \%$ ) and are separated by a point of zero transmission at $2.1 \mathrm{~cm}$, where the phase is undefined. This phase singularity could be attributed to the enhancement of a Wood anomaly triggered by the metal-dielectric interface. Moreover, a new transmission peak appears at $2.93 \mathrm{~cm}$ (III) [Fig. 2(b)], where 65\% transmission is accompanied by a zero phase change. If, in addition to the dielectric substrate, a superstrate of the same thickness and permittivity is introduced [Fig. 2(c)], peaks I and II are no longer visible, peak III increases in magnitude reaching $90 \%$, and the phase at the maximum is again zero rendering the structure virtually invisible. This is a behavior typical of Breit-Wigner resonances, ${ }^{20}$ which can be understood by noticing that Eq. (2) becomes a Lorentzian near the transmission maximum, the phase of which changes sign when the real part of the denominator vanishes (i.e., right on resonance), while the imaginary part evolves smoothly.

The more complicated spectral shape observed for the structures of Figs. 2(b) and 2(c), results from a complex interplay between hole polarizabilities, lattice sums, and guided modes in the dielectric slabs, which is well accounted for by our analytical theory (dashed curves). While the interaction between holes in the self-standing film (making up the $G_{\|}$lattice sum) has the same distance scaling as the dipoledipole interaction in free space, in the case of supported films the situation is qualitatively different, due to the existence of cutoff-free guided TM surface modes in the dielectric. These surfacelike waves trigger a slower decay with distance of the 
interhole interaction. Furthermore, hole-mediated coupling of the external light to the TM modes provides a loss channel that results in significant broadening of peak III, with absorption in the dielectric adding an insignificant contribution.

In conclusion, we have demonstrated, theoretically and experimentally, enhanced transmission of microwaves through quasiperiodic hole arrays in perfect conductors which cannot support surface plasmons. A direct relation between the reciprocal space maxima and the transmission peaks has been established. In particular, an invisible metal state characteristic of Breit-Wigner resonances has been observed, in which almost total transmission with zero phase change can be achieved by placing a structured film between two dielectric slabs. The wavelength position of the total transmission can be tuned either by varying the permittivity of the dielectric slabs or by appropriately scaling the pattern. In fact, we have already shown that this design is widely scalable and exhibits extraordinary transmission down to the telecom spectral region. ${ }^{12,13}$ Furthermore, the results presented here are almost independent of the polarization of the incident wave, due to the high orientational order of the quasicrystal. These characteristics are much desired in practical applications and we expect that such structures can prove useful over a wide region of the electromagnetic spectrum.

The authors acknowledge financial support from the Engineering and Physical Sciences Research Council, U.K.

${ }^{1}$ H. A. Bethe, Phys. Rev. 66, 163 (1944).

${ }^{2}$ C. C. Chen, IEEE Trans. Microwave Theory Tech. 19, 475 (1971).

${ }^{3}$ T. W. Ebbesen, H. J. Lezec, H. F. Ghaemi, T. Thio, and P. A. Wolff, Nature (London) 391, 667 (1998).

${ }^{4}$ H. F. Ghaemi, T. Thio, D. E. Grupp, T. W. Ebbesen, and H. J. Lezec, Phys.
Rev. B 58, 6779 (1998).

${ }^{5}$ L. Martín-Moreno, F. J. García-Vidal, H. J. Lezec, K. M. Pellerin, T. Thio, J. B. Pendry, and T. W. Ebbesen, Phys. Rev. Lett. 86, 1114 (2001).

${ }^{6}$ M. M. J. Treacy, Appl. Phys. Lett. 75, 606 (1999).

${ }^{7}$ J. Gómez-Rivas, C. Schotsch, P. Haring Bolivar, and H. Kurz, Phys. Rev. B 68, 201306(R) (2003).

${ }^{8}$ H. Cao and A. Nahata, Opt. Express 12, 1004 (2004).

${ }^{9}$ H. E. Went, A. P. Hibbins, J. R. Sambles, C. R. Lawrence, and A. P. Crick, Appl. Phys. Lett. 77, 2789 (2000).

${ }^{10}$ V. Lomakin, C. Nan-Wei, and E. Michielssen, IEEE Antennas and Propagation Society International Symposium, 22-27 June 2003 (unpublished), Vol. 2, p. 821.

${ }^{11}$ M. Sun, J. Tian, Z.-Y. Li, B.-Y. Cheng, D.-Z. Zhang, A.-Z. Jin, and H.-F. Yang, Chin. Phys. Lett. 23, 486 (2006).

${ }^{12}$ A. S. Schwanecke, N. Papasimakis, V. A. Fedotov, F. Huang, Y. Cheng, F. J. García de Abajo, and N. I. Zheludev, OSA Nanophotonics Topical Meeting, April 2006 (unpublished), p. 2628.

${ }^{13}$ F. J. García de Abajo, Y. Cheng, V. A. Fedotov, N. Papasimakis, A. S. Schwanecke, and N. I. Zheludev, Photonic Metamaterials: From Random to Periodic, Grand Island, The Bahamas, 5-8 June 2006 (unpublished).

${ }^{14}$ F. Przybilla, C. Genet, and T. W. Ebbesen, Appl. Phys. Lett. 89, 121115 (2006).

${ }^{15}$ T. Matsui, A. Agrawal, A. Nahata, and Z. V. Vardeny, Nature (London) 446, 517 (2007)

${ }^{16}$ J. Bravo-Abad, A. I. Fernandez-Dominguez, F. J. García-Vidal, and L. Martín-Moreno, e-print arXiv:cond-mat/0606121.

${ }^{17}$ F. J. García de Abajo, J. J. Sáenz, I. Campillo, and J. S. Dolado, Opt. Express 14, 7 (2006).

${ }^{18}$ Long-range interaction between holes dictates the transmission resonances, so that local variations between neighboring holes can be overlooked in this coherent-phase approximation, in which the phase imposed by the external field drives the effective phase difference in the response of distant holes.

${ }^{19}$ We apply Eq. (2) to thick films by interpreting $\alpha$ as a tensor containing the polarizability of both sides of the film (Ref. 17).

${ }^{20}$ L. D. Landau and E. M. Lifshitz, Quantum Mechanics: Non-Relativistic Theory, 2nd edition (Pergamon Press, London, 1958), Vol. 3, Chap. 17-18. 